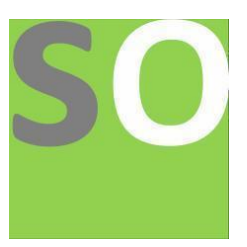

Article title: Ginsenoside Rg1 attenuates arsenic-induced mice nephrotoxicity via the activated HO-1/mTOR-associated apoptosis or autophagy signaling

Authors: yuan yang[1]

Affiliations: The First Affiliated Hospital of Hunan University of Medicine, Hunan University of Medicine[1]

Orcid ids: 0000-0002-2195-6138[1]

Contact e-mail: 807040461@qq.com

License information: This work has been published open access under Creative Commons Attribution License http://creativecommons.org/licenses/by/4.0/, which permits unrestricted use, distribution, and reproduction in any medium, provided the original work is properly cited. Conditions, terms of use and publishing policy can be found at https://www.scienceopen.com/.

Preprint statement: This article is a preprint and has not been peer-reviewed, under consideration and submitted to ScienceOpen Preprints for open peer review.

Funder: Natural Science Foundations of China

DOI: 10.14293/S2199-1006.1.SOR-.PPSYGZI.v1

Preprint first posted online: 17 May 2021

Keywords: Arsenic, Nephrotoxicity, Ginsenoside Rg1, Apoptosis, Autophagy 


\title{
Ginsenoside Rg1 attenuates arsenic-induced mice nephrotoxicity via the activated HO-1/mTOR-associated apoptosis or autophagy signaling
}

Yumei Tian ${ }^{\mathrm{a}}$, Feiyuan Zeng ${ }^{\mathrm{a}}$, Yuanyuan $\mathrm{Nie}^{\mathrm{a}}$, Rong $\mathrm{Chen}^{\mathrm{a}}$, Zhixi Lei ${ }^{\mathrm{a}}$, Changjun $\mathrm{Hu}^{\mathrm{a}}$, Peng Chen ${ }^{\mathrm{a}}$, Yuan Yang ${ }^{\mathrm{a}, \mathrm{b}, \mathrm{c}}{ }^{*}$, Huagui Chen ${ }^{\mathrm{a}}{ }^{*}$

\section{Authors' affiliations}

${ }^{a}$ Dong Medicine Key laboratory of Hunan Province, Department of laboratory medicine, School of Nursing, The First Affiliated Hospital of Hunan University of Medicine, Hunan University of Medicine, Huaihua 418000, Hunan, China

${ }^{b}$ Hunan Provincial Key Laboratory for Synthetic Biology of Traditional Chinese Medicine, Hunan University of Medicine, Huaihua 418000, Hunan, China

${ }^{\mathrm{c}}$ Hunan Province Key Laboratory for Antibody-based Drug and Intelligent Delivery System, Hunan University of Medicine, Huaihua 418000, Hunan, China

\begin{abstract}
All authors' email: Y. Tian (moldrains@163.com); F. Zeng (617905387@qq.com); Y. Nie (1878747063@qq.com); R. Chen (1278945877@qq.com); Z. Lei (2440794689@qq.com); C. Hu (hcj6713@126.com); P Chen (505984893@qq.com); Y. Yang (yang1977yuan@126.com); Huagui Chen (1226485848@qq.com)
\end{abstract}

\section{Corresponding author details}

* Corresponding author: Yuan Yang \& Huagui Chen, Dong Medicine Key laboratory of Hunan Province, Department of laboratory medicine, School of Nursing, The First Affiliated Hospital of Hunan University of Medicine, Hunan University of Medicine, Huaihua 418000, Hunan, China. E-mail addresses: yang1977yuan@126.com; 1226485848@qq.com

Running title: Ginsenoside Rg1 attenuates arsenic-induced nephrotoxicity 


\begin{abstract}
Nephrotoxicity attributed to environmental arsenic exposure, has been recognized by animal experiments and populational survey over 30 years in China, given a significance of public health by preventing from the disorder of renal function and hispathological abnormality. Here, Ginsenoside $\operatorname{Rg} 1$ (Rg1) as the commercial bioactive product of ginseng, play a beneficial role via antioxidant, anti-inflammatory and anti-apoptotic effects, which is poorly understood in arsenic-induced nephrotoxicity. The present study applied animal experiments to explore the pharmacological effects of Rg1 on sodium arsenite (SA)-induced nephrotoxicity in mice. Results showed that SA exposure led to renal pathological damage, and induced renal oxidative stress and the elevated levels of apoptosis or autophagy-associated indices in kidney. Further, western-blotting results confirmed the upregulations of pro-apoptotic Bax or autophagic unc-51-like kinase-1 (ULK1) or LC3-B signal, and the downregulations of HO-1 or mTOR signal and autophagy substrate sequestosome 1 (p62/SQSTM1) in kidney. Significantly, the intervention with Rg1 alleviated arsenic-induced renal pathological damage and oxidative stress, and upregulated the levels of HO-1, mTOR and p62, while the levels of Bax, ULK1 or LC3-B downregulated in kidney. In conclusion, the intervention with $\operatorname{Rg} 1$ relieves arsenic-induced mice nephrotoxicity maybe involved in the regulation of HO-1/mTOR-related apoptotic or autophagic signaling.
\end{abstract}

Keywords: Arsenic; Nephrotoxicity; Ginsenoside Rg1; Apoptosis; Autophagy

Running title: Ginsenoside Rg1 alleviates arsenic-induced nephrotoxicity 


\section{Introduction}

Kidney as the vital organ in body, exerting the functions of excreting, metabolism, enzymatic reaction, immunization etc, which is considered as one of arsenic toxic targets due to lifestyle pollution or environmental exposure. Over 30 years, arsenic pollution-associated nephrotoxicity has been recognized as the disorder of renal function and hispathological abnormality of glomerulus or renal tubule in kidney, which is consistently concerned in Guizhou Province of China [1-2]. Mechanistically, Arsenic was reported as the inducers of oxidative stress-associated apoptosis and autophagy in osteosarcoma or in chickens, associated with the suppression of protein kinase $\mathrm{B}(\mathrm{Akt} / \mathrm{PKB})$ or mammalian target of rapamycin (mTOR) signaling pathways, characterized as the increased levels of autophagy markers such as microtubule associated protein light chains 3-II (LC3- II ), Beclin-1, while could be significantly attenuated by ROS scavenger [3-4]. At present, a study indicates that bioactive polysaccharides extract of traditional Chinese medicine Ginseng can alleviate effectively renal ischemia/reperfusion (I/R) injury by its anti-oxidative effects [5], while the pharmacological effects on arsenic induced nephrotoxicity is poorly understood.

Ginseng, full named by Panax ginseng C. A. Mey, a perennial herb of the genus aconitiaceae, and its polysaccharides Ginsenoside $\operatorname{Rg} 1(\mathrm{Rg} 1)$ as the primary active substance, which has been applied as the commercial product of ginseng. Many studies showed the significant effects of Rg1 on vascular injury, hepatic or glomerular fibrosis, ischemic cardiovascular diseases, neurotoxicity, etc. For example, $\operatorname{Rg} 1$ 
antagonizes oxidative stress by increasing the levels of total superoxide dismutase (T-SOD), catalase (CAT), or glutathione (GSH) levels to exert its cardioprotective effect [6]; Rg1 decreases apoptosis and activates PI3K/Akt pathway against lipopolysaccharide (LPS)-induced human renal tubular epithelial cells HK-2 injury [7]; Rg1 alleviate angiotensin II-induced podocyte injury by inhibiting renal podocyte autophagy via the activation of AMP activated protein kinase (AMPK)/mTOR pathway [8]; Rg1 reduced autophagy in rat renal tubular NRK-52E cells exposed to aldosterone by decreasing intracellular ROS and preserving mTOR activity [9]. Here, the present study explored the role of $\mathrm{Rg} 1$ intervention on Sodium arsenite (SA) induced mice nephrotoxicity.

\section{Materials and methods}

\subsection{Experimental design.}

The protocol for this study was approved by Ethics Committee of Hunan University of Medicine (no.1907006). 32 healthy C57BL/6 male mice were purchased from Hunan Slack King of Laboratory Animal Co., Ltd (Changsha, China) [Laboratory Animal Certificate: SCXK2019-0004]. The mice weight as $25.2 \pm 1.1 \mathrm{~g}$, and ranging in age from 9 to 10 weeks. After one week of adaptive feeding with standard temperature $\left(20-22^{\circ} \mathrm{C}\right)$ and relative humidity (60-70\%) under a 12-hour (h) light/dark cycle, the mice were divided into the following 4 groups ( $n=8 /$ group): I ) Control group: drink deionized water; II ) Single SA treatment group: $\mathrm{NaAsO}_{2}$ (CAS.7784-46-5, Sigma-Aldrich, USA) was treated by gavage $10.0 \mathrm{mg} / \mathrm{kg}$ (dissolved in deionized water by $1.0 \mathrm{mg} / \mathrm{ml}$, dose: $10.0 \mathrm{mg} / \mathrm{kg} . \mathrm{bw}, 0.25 \mathrm{ml} / \mathrm{time})$, once every 
other day for 2 weeks, referred to the previous studies [10-11]; III) $\operatorname{Rg} 1+\mathrm{SA}$ treatment group: Rg1 (CAS:22427-39-0, Duma Biotechnology Co., Ltd., China) was treated by intraperitoneal injection (dose: $20.0 \mathrm{mg} / \mathrm{kg} . \mathrm{bw}$ ) 8 hours in advance of SA treatment, referred to Gao Y et al' study [12-13]; IV) Single Rg1 treatment group: $\operatorname{Rg} 1$ was diluted by deionized water to $20.0 \mathrm{mg} / \mathrm{kg} . \mathrm{bw}, 0.15 \mathrm{ml} /$ time, once every other day for 14 days. At the end of the experiment, mice were anesthetized and euthanized by $0.9 \%$ sodium pentobarbital intraperitoneal injection, then the blood samples was collected immediately, and renal tissues were islated for the further analysis.

\subsection{Kidney function and pathological evaluation.}

For the evaluation of kidney function, following blood samples were obtained from posterior orbital venous plexus, the samples were centrifuged at $4^{\circ} \mathrm{C}$ and $400 \times g$ for 5 min, and the supernatant was applied to measure serum creatinine (Scr) according to the instructions of Elisa assay kit (DM651612, Duma Biotechnology Co., Ltd., China), then renal tissue sections were prepared for pathological evaluation. Detailedly, kidney samples were fixed with $4 \%$ paraformaldehyde for $72 \mathrm{~h}$, then embedded in paraffin for preparing $5 \mu \mathrm{m}$ pathological sections, and hematoxylin-eosin (HE) staining were performed for evaluating the pathological characteristics of kidney tissues under Motic microscope (DMB5-2231P1, x100), and the score of tubular injury (TI) in kidney was assigned referring to our previously method [14]. Further, the paraffin-embedded tissue sections were used to detect DNA fragmentation of renal cells apoptosis in situ by TUNEL apoptosis assay Kit-HRP-DAB (ab206386, Abcam) according to the manufacturer's instructions. Following the addition of biotin-labeled 
deoxynucleotides-biotinylated nucleotides and binding with a streptavidin-horseradish peroxidase (HRP) conjugate-diaminobenzidine (DAB), and counterstaining with methyl green, the insoluble brown substrate was generated at the site of DNA fragmentation in apoptotic cells, used to identify apoptotic cells. The apoptotic cells were calculated using Motic Virtual Microscope 1.0, and 10 fields of high power vision (magnification, $\mathrm{x} 200$ ) were selected randomly for evaluating the proportions of apoptotic cells in each slide.

\subsection{Measurement of oxidative stress in renal tissues.}

Following the isolated kidney tissues were weighed, Phosphate Buffered Saline (PBS) was added at the ratio of 1:9 (w/v) for homogenization by ULTRA-TURRAX homogenizer, then Roswell Park Memorial Institute (RPMI) medium (containing $0.05 \%$ type II collagenase, $0.002 \%$ DNase I and $0.6 \%$ bovine) was added for incubating at $37^{\circ} \mathrm{C}$ for $30 \mathrm{~min}$, and 3 freeze-thaw cycles were performed for Lysing cells. Finally, the tissue homogenates were centrifugated at $4^{\circ} \mathrm{C}, 1500 \times g$ for $10 \mathrm{~min}$, and the supernatants were collected to evaluate the levels of oxidative stress indices GSH and Malondialdehyde (MDA) by ELISA assay kit (GSH: MLGR-E20324; MDA: MLGR-E20206, Shanghai Mlbio Biotechnology Co., Ltd.) according to the manufacturer's instructions, respectively. The above samples were measured by Microplate reader (BioTek800 TS, USA) at wavelength of $450 \mathrm{~nm}$, and was converted from optical density (OD) values to the contents in per gram renal tissue according to a standard curve. 


\subsection{Immunohistochemical evaluation in kidney tissues.}

For the evaluation of positive percentage of apoptosis or autophagy indices Caspase-3, Beclin-1 protein in kidney tissues, the method of immunohistochemical (IHC) staining was performed referred to our previous study [15]. Briefly, sections embedded in paraffin were dewaxed by xylene and the graded alcohol, and the step of antigen retrieval and sections washing were performed, sections were then incubated at $4^{\circ} \mathrm{C}$ overnight with primary polyclonal antibody Caspase-3 (ab32499, abcam), Beclin-1 (ab62557, abcam), respectively. Subsequently, sections were washed with PBS and incubated with polymeric HRP-labeled secondary antibody (sp9000, Zsbio Biotechnology Co., Ltd., China) at room temperature for $10 \mathrm{~min}$. Finally, the coloration step was performed by temporarily prepared 3, 3-diaminobenzidine (DAB) solution, and the percentages of IHC-positive cells were measured by calculating 10 fields under high power vision (magnification, x200).

\subsection{Immunofluorescence detection of autophagy biomarker LC3-B.}

Immunofluorescence of autophagy biomarker LC3-B was determined referred to Maghames et al' method with some modifications [16]. Briefly, renal tissues were isolated and fixed in $4 \%$ paraformaldehyde at $4{ }^{\circ} \mathrm{C}$ overnight, and sections of $5 \mu \mathrm{m}$ were prepared, then dewaxed, hydrated, and washed by PBS. Following the sections were performed by the methods of high-pressure antigens repair and goat serum blocking. The sections were incubated with the primary anti-LC3-B antibody $(1: 100$ in PBS) (ab51520, Abcam) at $4{ }^{\circ} \mathrm{C}$ overnight, were then washed three times with PBS for 5 min each time, then incubated with the secondary antibody Alexa Fluor ${ }^{\circledR} 555$ 
Goat Anti-Rabbit IgG (ab150078, Abcam) at $37^{\circ} \mathrm{C}$ for $40 \mathrm{~min}$, then washed three times with PBS and were counterstained by 4',6-diamidino-2-phenylindole (DAPI) dilactate staining at room temperature for $5 \mathrm{~min}$, then following sections were washed with PBS, fluorescence was observed by a confocal microscope (x400, LeicaTCS-SP5), with emission wavelength of $535 \mathrm{~nm}$ for Alexa Fluor ${ }^{\circledR} 555$, or $340 \mathrm{~nm}$ for DAPI.

\subsection{Western-Blotting analysis of kidney tissues.}

Following the total of proteins were extracted from the homogenized $(10 \% \mathrm{w} / \mathrm{v})$ kidney tissues lysates using radioimmunoprecipitation assay (RIPA) buffer (BioVision Inc., USA). Protein concentration was determined using the Bradford reagent (Sigma-Aldrich, USA), then the expressional levels of heme oxygenase-1 (HO-1), mTOR, pro-apoptotic Bcl2-Associated X Protein (Bax) and autophagy influx in kidney were detected by the method of Western-Blotting. Briefly, the steps of sodium dodecyl sulfate-polyacrylamide gel electrophoresis (SDS-PAGE) and transferring onto polyvinylidine membranes were performed. Subsequently, after blocking non-specific binding sites, the membranes were incubated for $16 \mathrm{~h}$ at $4{ }^{\circ} \mathrm{C}$ with the following primary antibodies: Rabbit monoclonal HO-1 antibody (ab68477, Abcam); Rabbit polyclonal mTOR antibody (ABIN6263424, Abnova); Rabbit monoclonal Bax antibody (ab182733, Abcam); Rabbit polyclonal unc-51-like kinase-1 (ULK1) antibody (ab167139, Abcam); Rabbit polyclonal SQSTM1/p62 antibody (ab155686, Abcam); Rabbit polyclonal Anti-LC3A/B antibody (ab128025, Abcam). And, anti-rabbit IgG-HRP Secondary Antibody (LS-C86382, LSBio, USA) were incubated 
for $2 \mathrm{~h}$ at room temperature and imaged by enhanced chemiluminescence reagent (Beyotime, China). The relative level of the target protein expression is evaluated by the integrated absorbance (IA) value ratio of target protein vs internal control anti- $\beta$-actin polyclonal antibody (ab6276, abcam).

\subsection{Statistical Analysis.}

Data were expressed as mean \pm standard deviation (SD) and was analyzed by SPSS software (version 23.0; IBM Corp). Evaluation of the difference between groups was performed by one-way analysis of variance (ANOVA) test with $\mathrm{T}$ test; Data of TUNEL or IHC experiment were expressed as the percentage (\%), and was analyzed by $\chi^{2}$ test. $P<0.05$ was considered as the standard of significant difference between groups.

\section{Results}

\subsection{Arsenic induced renal damage was alleviated by $\mathrm{Rg} 1$}

As presented in Figure 1A, HE staining of kidney sections showed renal histopathological injury in SA exposure group, characterized as the dilated and congested glomerular capillaries, the balloon enlarged, and irregular tubular lumen, edema tubular epithelial cells or renal interstitial, some local nucleus disappear and necrosis. While the intervention with $\operatorname{Rg} 1$ showed an amelioration of kidney pathological injury such as the alleviations of glomerular capillaries congestion, tubular epithelial cells or renal interstitial edema compared with SA exposure group. As presented in Figure 1B, compared to control group, a significant increase in serum 
creatinine or TI score was showed in SA exposure group $(P<0.05)$, while the intervention with $\operatorname{Rg} 1$ pretreatment showed a downregulation of serum creatinine or TI score compared with that of SA exposure group, indicating that the pretreatment with $\mathrm{Rg} 1$ was protective against SA-induced renal injury, more details were in Supplemental Table S1.

\subsection{Rg1 downregulated arsenic-induced apoptosis or autophagy indices in kidney}

As shown in Figure 2A, the TUNEL experiments showed SA exposure led to an increased TUNEL positive cells in kidney compared with that of control group, while the intervention with $\mathrm{Rg} 1$ resulted in a significant reduce of apoptosis compared with that of SA exposure group $(P<0.05)$, representative images were presented as Figure 2B. As shown in Figure 2C, the $\mathrm{IHC}$ experiments showed the increased positive propotion of Beclin-1 or Caspase-3 in renal tissues compared with that of control group, while the intervention with $\mathrm{Rg} 1$ showed a significant downregulation as comparison with that of SA exposure group $(P<0.05)$, details were presented in Supplemental Figure S1-2 \& Table S1. Similar to IHC results of Beclin-1, the immunofluorescence experiment of autophagy biomarker LC3-B showed the enhanced staining in SA exposure group, while was ameliorated by $\mathrm{Rg} 1$ intervention (Figure 2D), indicating that the upregulated autophagy induced by SA was ameliorated by the intervention with $\mathrm{Rg} 1$.

\section{3. $R g 1$ improved arsenic-induced oxidative stress in kidney}

As shown in Figure 3, compared with control group, SA exposure led to a decreased 
GSH and an increased MDA in kidney $(P<0.05)$, indicating that SA induced the activated oxidative stress in kidney. After the intervention with $\operatorname{Rg} 1$, resulting in a significant recovery in GSH and a significant downregulation in MDA $(P<0.05)$, indicating that the antioxidative role of $\operatorname{Rg} 1$ antagonizing against arsenic-induced oxidative stress, details as shown in Supplemental Table S2.

\subsection{Rgl activated HO-1 or mTOR signals while inhibited autophagy influx.}

The levels of HO-1, mTOR and apoptosis or autophagy-related Bax, SQSTM1/p62, ULK1 or LC3-A/B in kidney were measured by immunoblotting method. As shown in Figure 4A, B, or C, a significant downregulation of HO-1, mTOR and SQSTM1/p62 while a significant upregulation of Bax, ULK1 and LC3-B in renal tissues were found in SA exposure group $(P<0.05)$, indicating that SA inhibited anti-oxidative signaling and activated apoptosis or autophagy influx in kidney. Interestingly, compared with SA exposure group, the intervention with Rg1 upregulated significantly the levels of HO-1, mTOR and SQSTM1/p62, and downregulated the levels of Bax, ULK1 or LC3-B in kidney $(P<0.05)$, indicating that Rgl activated anti-oxidant HO-1 or mTOR signals and led to the inhibitions of pro-apoptotic Bax and autophagy influx in kidney maybe contributed to the renoprotection against SA-induced nephrotoxicity, details as shown in Supplemental material (Figure S3 4\&Table S3).

\section{Discussion}

Ginseng as a traditional herb recognized as Panax ginseng in China, Korea, Japan, or identified as Panax quinquefolius in America, its bioactive extract is refined from the 
root of Ginseng, which are steroidal saponins conjugated to different sugar moieties and polysaccharides accounted for $10 \sim 20 \%$ of total weight, named as Ginseng Polysaccharides or ginsenosides [17]. Rg1 as the commerced Ginsenoside Polysaccharides, play a beneficial role in a wide range of injuries or diseases by its activities of anti-oxidant, anti-inflammatory, antidepressant, antitumor and immune-regulatory properties both in vitro and in vivo [18-19]. For example, some studies found that Rg1 can activate Akt signaling and the phosphorylated mTOR signaling [20], and resulted in the amelioration of cell apoptosis and autophagy, contributing to the amelioration of rat ischemia/reperfusion (I/R) cardiac injury [21], and Rg1 shows its anti-apoptosis functions involved in the regulation of mitochondrial apoptosis pathway-mediated B-cell lymphoma-2 (Bcl-2), Bcl-2-associated X protein (Bax), and caspase-3 [22]. In particular, Rg1 ameliorates renal fibrosis involved in the regulation of the Klotho/TGF- $\beta 1 /$ Smad signaling pathway in rats with obstructive nephropathy [23]; The renoprotection mechanism of Rg1 in a mouse subacute damage model is attributed to alleviate oxidative stress injury in kidney [24]. In our study, the intervention with $\operatorname{Rg} 1$ showed a significant renoprotective role by the attenuations of oxidative stress, apoptosis and autophagy influx in kidney compared with SA exposure group, characterized as the upregulations of antioxidants GSH, HO-1 or mTOR signals, while the downregulations of pro-apoptotic Caspase-3, Bax, and autophagy-related biomarkers Beclin-1, ULK1, or LC3-B in kidney. This results indicate Rg1 maybe prevent from arsenic-induced renal 
injury via the activation of HO-1 or mTOR signaling and the inhibitions of apoptosis signal and autophagy influx in kidney.

mTOR is the intracellular serine or threonine kinase survival signaling pathway in body, which is associated closely with anti-oxidant or autophagy influx. Some studies found HO-1 is associated closely with mTOR signaling pathway. For example, HO-1/emopamil-binding protein (EBP) interaction alleviates cholesterol-induced hypoxia through the activation of the Nrf2/mTOR pathways [25]; The upregulation of HO-1 is accompanied by activation of mTOR signaling in ethanol-treated human oesophageal squamous carcinoma cells [26]. Further, some studies revealed the inhibition of mTOR protein activity can lead to the downregulated phosphorylation levels of its downstream target protein $\mathrm{p} 70 \mathrm{~S} 6 \mathrm{~K}$ and eukaryotic initiation factor $4 \mathrm{E}$ binding protein 1 (4E-BP1), resulting in reduced gene-protein translation, thereby inhibiting cell proliferation and activation of autophagy [27], and the suppression of the PI3K/AKT/mTOR signaling pathway accelerates chondrocytes apoptosis and autophagy in mice model of rheumatoid arthritis (RA) treated by Artesunate [28]. Conversely, the activation of mTOR signaling can prevent ULK1 (a homologue of yeast ATG1) activation by phosphorylating Ulk1 Ser 757, and disrupting the interaction between ULK1 and AMPK, thus lead to the inhibition of autophagy [29], and the activation of $\mathrm{Nrf2} / \mathrm{HO}-1$ pathway was associated with the up-regulation of HIF $1 \alpha$ and the downregulation of hypoxia-aroused H9c2 cell apoptosis [30].

Enlightened on the above studies, in our study, the results of the intervention with $\mathrm{Rg} 1$ exerts the activation of antioxidant enzyme HO-1 and mTOR signaling, led 
to the downregulations of autophagy- or apoptosis-related indices in kidney. So it is speculated that $\mathrm{Rg} 1$ induces the activation of HO-1 signaling, further activates the mTOR signaling pathway leads to the activation of intracellular serine/threonine kinase and the transformation of $\mathrm{G}$ to $\mathrm{S}$ phase in cell cycle for cell proliferation, thus leading to the inhibition of apoptosis or autophagy influx, contributing to the alleviation of renal injury induced by arsenic.

Furthermore, the results of elevated GSH and downregulated MDA indicate that Rg1 alleviates arsenic-induced renal oxidative damage, maybe involved in its regulation of renoprotection mechanism. Combined with the close association between antioxidant GSH and the characteristics of apoptosis or autophagy-related signals during arsenic toxicity [31-32]. The present study indicates that Rg1 elicited the elevated levels of HO-1 and GSH in cells, further promote the activation of anti-apoptotic Bcl-2 and the inhibition of pro-apoptotic Bax signal, resulting in the alleviations of renal apoptosis, contributing to the renoprotection against arsenic-induced renal injury in mice.

In conclusion, $\operatorname{Rg} 1$ antagonizes effectively against arsenic-induced kidney injury in mice, maybe involved in the regulation of antioxidative HO-1 and mTOR pathway, leading to the inhibitions of autophagy influx or apoptosis. However, it is still necessary for further exploring the renoprotective role of $\mathrm{Rg} 1$ in arsenic-induced nephrotoxicity, including the upstream PI3K/Akt signaling and the downstream p70S6k, S6K1 or 4E-BP1 signaling in mTOR pathway, and explored endoplasmic reticulum-related CCAAT/enhancer binding protein homologous protein $(\mathrm{CHOP})$ 
signaling, or mitochondria-mediated inflammatory signaling or autophagy-related AMPK signaling pathway to further reveal their interaction mechanism contributed to prevent from renal injury, providing the scientific basis of the antagonistic mechanism elicited by Rg1 against arsenic-induced nephrotoxicity.

\section{References}

[1] 杨运旗, 孙兰英, 黄晓欣, 张爱华, \& 陈宁. (2003). 慢性燃煤砷污染所致肾损害的临 床病理观察. 中华地方病学杂志, 22(004), 358-359. YANG Yun-qi, SUN Lan-ying, HUANG Xiao-xin, ZHANG Ai-hua, CHEN Ning. The clinically pathologic investigation of renal function impairment by aresnism caused by coal-burning pollution, Chinese Journal of Endemiology, 2003, 22(4): 358-9. DOI : 10.3760/cma.j.issn.1000-4955.2003.04.025

[2] 洪峰，张爱华，\& 金泰庴. (2007). 燃煤砷污染地区砷中毒患者的肾功能损害. 环境 与职业医学, 024(006), 580-583. Hong Feng, Zhang Aihua, Jin Taiyi. A Study on Renal Dysfunction ofArsenic Poisoning Victims Caused by Air Pollution from Coal Burning, $J$ Environ Occup Med , 2007, 24(6): 580-3 (Article in Chinese). DOI : 10.3969/j.issn.1006-3617.2007.06.003

[3] Wang G, Zhang T, Sun W, Wang H, Yin F, Wang Z, Zuo D, Sun M, Zhou Z, Lin B, Xu J, Hua Y, Li H, Cai Z. Arsenic sulfide induces apoptosis and autophagy through the activation of $\mathrm{ROS} / \mathrm{JNK}$ and suppression of Akt/mTOR signaling pathways in osteosarcoma, Free radical biology \& medicine, 2017, 106: 24-37. doi: 10.1016/j.freeradbiomed.2017.02.015.

[4] Shao YZ, Zhao HJ, Wang Y, Liu JJ, Li JL, Luo LY, Xing MW. The apoptosis in arsenic-induced oxidative stress is associated with autophagy in the testis tissues of chicken, Poultry science, 2018, 97(9): 3248-3257. doi: 10.3382/ps/pey156.

[5] Liu Z, Li C, Zhang Q, Tao M. Effect of Renshen polysaccharides on oxidative injury in kidney IR rabbits, Carbohydrate polymers, 2012, 90(2):773-777. doi: 10.1016/j.carbpol.2012.05.040.

[6] Zhu D, Wu L, Li CR, Wang XW, Ma YJ, Zhong ZY, Zhao HB, Cui J, Xun SF, Huang XL, Zhou Z, Wang SQ. Ginsenoside Rg1 protects rat cardiomyocyte from hypoxia/reoxygenation oxidative injury via antioxidant and intracellular calcium homeostasis, Journal of cellular biochemistry, 2009, 108(1):117-124. doi: $10.1002 / \mathrm{jcb} .22233$.

[7] Ni XJ, Xu ZQ, Jin H, Zheng SL, Cai Y, Wang JJ. Ginsenoside Rg1 protects human renal tubular epithelial cells from lipopolysaccharide-induced apoptosis and inflammation damage, Brazilian journal of medical and biological research, 2017, 51(2): e6611. doi: 10.1590/1414-431X20176611.

[8] Mao N, Tan RZ, Wang SQ, Wei C, Shi XL, Fan JM, Wang L. Ginsenoside Rg1 inhibits 
angiotensin II-induced podocyte autophagy via AMPK/mTOR/PI3K pathway, Cell biology international, 2016, 40(8):917-925. doi: 10.1002/cbin.10634.

[9] Wang L, Mao N, Tan RZ, Wang HL, Wen J, Liu YH, Furhad M, Fan JM. Ginsenoside Rg1 reduces aldosterone-induced autophagy via the AMPK/mTOR pathway in NRK-52E cells, International journal of molecular medicine, 2015, 36(2):518-526. doi: 10.3892/ijmm.2015.2242.

[10] Turk E, Kandemir FM, Yildirim S, Caglayan C, Kucukler S, Kuzu M. Protective Effect of Hesperidin on Sodium Arsenite-Induced Nephrotoxicity and Hepatotoxicity in Rats, Biol Trace Elem Res, 2019, 189(1):95-108. doi: 10.1007/s12011-018-1443-6.

[11] Bashir S, Sharma Y, Irshad M, Gupta SD, Dogra TD. Arsenic-induced cell death in liver and brain of experimental rats, Basic \& clinical pharmacology \& toxicology, 2006, 98(1):38-43. doi: 10.1111/j.1742-7843.2006.pto_170.x.

[12] Gao Y, Li J, Chu S, Zhang Z, Chen N, Li L, Zhang L. Ginsenoside Rg1 protects mice against streptozotocin-induced type 1 diabetic by modulating the NLRP3 and Keap1/Nrf2/HO-1 pathways, European journal of pharmacology, 2020, 866:172801. doi: 10.1016/j.ejphar.2019.172801.

[13] Qin Q, Lin N, Huang H, Zhang X, Cao X, Wang Y, Li P. Ginsenoside Rg1 ameliorates cardiac oxidative stress and inflammation in streptozotocin-induced diabetic rats, Diabetes, metabolic syndrome and obesity : targets and therapy, 2019, 12:1091-1103. doi: 10.2147/DMSO.S208989.

[14] Song MF, Yang Y, Yi ZW, Zhang ZQ, Shen XD, Hu GH, Zhu YF. Sema 3A as a biomarker of the activated mTOR pathway during hexavalent chromium-induced acute kidney injury. Toxicol Lett. 2018, 299:226-235. doi: 10.1016/j.toxlet.2018.09.005.

[15] Yuan Y, Guo-Qing P, Yan T, Hong-Lin Y, Gong-Hua H, Cai-Gao Z. A study of PKM2, PFK-1, and ANT1 expressions in cervical biopsy tissues in China, Medical oncology (Northwood, London, England), 2012, 29(4):2904-2910. doi: 10.1007/s12032-011-0154-z.

[16] Maghames CM, Lobato-Gil S, Perrin A, Trauchessec H, Rodriguez MS, Urbach S, Marin $\mathrm{P}$, Xirodimas DP. NEDDylation promotes nuclear protein aggregation and protects the Ubiquitin Proteasome System upon proteotoxic stress, Nature communications, 2018, 9(1): 4376. doi: 10.1038/s41467-018-06365-0.

[17] Jiao R, Liu Y, Gao H, Xiao J, So KF. The Anti-Oxidant and Antitumor Properties of Plant Polysaccharides, The American journal of Chinese medicine, 2016, 44(3):463-488. doi: 10.1142/S0192415X16500269.

[18] Sun Y. Structure and biological activities of the polysaccharides from the leaves, roots and fruits of Panax ginseng C.A. Meyer: An overview, Carbohydrate polymers, 2011, 85(3):490-499. DOI: 10.1016/j.carbpol.2011.03.033

[19] Xiong X, Huang G, Huang H. The antioxidant activities of phosphorylated polysaccharide from native ginseng, International journal of biological macromolecules, 2019, 126:842-845. doi: 10.1016/j.ijbiomac.2018.12.266.

[20] Li F, Li X, Peng X, Sun L, Jia S, Wang P, Ma S, Zhao H, Yu Q, Huo H. Ginsenoside $\mathrm{Rg} 1$ prevents starvation-induced muscle protein degradation via regulation of $\mathrm{AKT} / \mathrm{mTOR} / \mathrm{FoxO}$ signaling in $\mathrm{C} 2 \mathrm{C} 12$ myotubes, Experimental and therapeutic medicine, 2017, 14(2):1241-1247. doi: 10.3892/etm.2017.4615. 
[21] Qin L, Fan S, Jia R, Liu Y. Ginsenoside Rg1 protects cardiomyocytes from hypoxia-induced injury through the PI3K/AKT/mTOR pathway, Die Pharmazie, 2018, 73(6):349-355. doi: 10.1691/ph.2018.8329.

[22] Zhou Y, Wang YP, He YH, Ding JC. Ginsenoside Rg1 Performs Anti-Aging Functions by Suppressing Mitochondrial Pathway-Mediated Apoptosis and Activating Sirtuin 3 (SIRT3)/Superoxide Dismutase 2 (SOD2) Pathway in Sca- ${ }^{+}$HSC/HPC Cells of an Aging Rat Model, Medical science monitor, 2020, 26: e920666. DOI: 10.12659/MSM.920666

[23] Li SS, He AL, Deng ZY, Liu QF. Ginsenoside-Rg1 Protects against Renal Fibrosis by Regulating the Klotho/TGF- $\beta 1 /$ Smad Signaling Pathway in Rats with Obstructive Nephropathy, Biological \& pharmaceutical bulletin, 2018, 41(4):585-591. doi: 10.1248/bpb.b17-00934.

[24] Fan Y, Xia J, Jia D, Zhang M, Zhang Y, Huang G, Wang Y. Mechanism of ginsenoside $\mathrm{Rg} 1$ renal protection in a mouse model of d-galactose-induced subacute damage. Pharm Biol. 2016, 54(9):1815-21. doi: 10.3109/13880209.2015.1129543.

[25] Jin X, Xu Z, Cao J, Yan R, Xu R, Ran R, Ma Y, Cai W, Fan R, Zhang Y, Zhou X, Li Y. HO-1/EBP interaction alleviates cholesterol-induced hypoxia through the activation of the AKT and Nrf2/mTOR pathways and inhibition of carbohydrate metabolism in cardiomyocytes. Int J Mol Med. 2017, 39(6):1409-1420. doi: 10.3892/ijmm.2017.2979.

[26] Hu JL, Xiao L, Li ZY, Wang Q, Chang Y, Jin Y. Upregulation of HO-1 is accompanied by activation of p38MAPK and mTOR in human oesophageal squamous carcinoma cells. Cell Biol Int. 2013, 37(6):584-92. doi: 10.1002/cbin.10075.

[27] Wang Y, Zhang H. Regulation of Autophagy by mTOR Signaling Pathway, Advances in experimental medicine and biology, 2019, 1206: 67-83. doi: 10.1007/978-981-15-0602-4_3.

[28] Feng FB, Qiu HY. Effects of Artesunate on chondrocyte proliferation, apoptosis and autophagy through the PI $3 \mathrm{~K} / \mathrm{AKT} / \mathrm{mTOR}$ signaling pathway in rat models with rheumatoid arthritis. Biomed Pharmacother, 2018, 102:1209-1220. doi: 10.1016/j.biopha.2018.03.142.

[29] Kim J, Kundu M, Viollet B, Guan KL. AMPK and mTOR regulate autophagy through direct phosphorylation of Ulk1, Nature Cell Biology, 2011, 13(2):132-141. doi: $10.1038 / \mathrm{ncb} 2152$.

[30] $\mathrm{Yu} \mathrm{H}$, Chen B, Ren Q. Baicalin relieves hypoxia-aroused H9c2 cell apoptosis by activating Nrf2/HO-1-mediated HIF1 $\alpha /$ BNIP3 pathway. Artif Cells Nanomed Biotechnol. 2019, 47(1):3657-3663. doi: 10.1080/21691401.2019.1657879.

[31] Huang YC, Yu HS, Chai CY. Roles of oxidative stress and the ERK1/2, PTEN and p70S6K signaling pathways in arsenite-induced autophagy, Toxicol Lett, 2015, 239(3): 172-181. doi: 10.1016/j.toxlet.2015.09.022.

[32] Zhao H, Wang Y, Liu J, Guo M, Fei D, Yu H, Xing M. The cardiotoxicity of the common carp (Cyprinus carpio) exposed to environmentally relevant concentrations of arsenic and subsequently relieved by zinc supplementation, Environmental pollution (Barking, Essex : 1987), 2019, 253:741-748. doi: 10.1016/j.envpol.2019.07.065. 


\section{Authors' contributions}

Y.Y., and C.H., Study design, wrote this original manuscript; Y.N., R.C., Z.L., C. H., P.C., Performed the entire experiments; Y.T., and F. Z., experimental data analysis and visualization

\section{Ethics approval and consent to participate}

This study was approved by the Medical Ethics Committee of Hunan University of Medicine (no.1907006), and was performed according to the guidelines of the Ethics of the World Medical Association (Declaration of Helsinki). Informed consent was therefore not required.

\section{Availability of data and materials}

All data generated or analyzed in this study are included in this article and supplemental material, additional data related to this paper may be available from the corresponding author on reasonable request.

\section{Competing interests}

The authors declare that they have no competing interests.

\section{Acknowledgements}

This work was supported by Natural Science Foundations of China (grant nos. 81960597), innovation team of laboratory medicine, school of nursing, Hunan University of Medicine (2019-48), scientific research project of hunan health committee (20200073), demonstration base of medical emergency treatment of Hunan Province (2020SK4020). 


\section{Figures legends}

Figure 1. Characterisrics of renal function or histopathology following SA treatment or $\operatorname{Rg} 1$ intervention in mice

A: Histogram of serum creatinine (Scr) content after SA treatment or Rg1 intervention: SA treatment showed the increased level of Scr, which was downregulated after Rg1 intervention;

B: Representative histopathological images (HE, x100) of renal tissues sections. (I) Control group: kidney histology was normal, tubular epithelial cells showed smooth nuclear membrane, uniform cytoplasmic staining, normal nucleoplasmic ratio, and normal cell-to-cell spacing (as shown by the black arrow); (II) SA treatment group: Mice were administrated orally by SA at $10.0 \mathrm{mg} / \mathrm{kg} . \mathrm{bw}$ once every other day for 14 days, renal tissues showed the Glomerular balloon enlargement, capillary hyperemia, irregular renal tubule lumen, tubule epithelial cell edema, cell nucleus disappearance in some places (as shown by the black arrow); (III) Rg1+SA treatment group: Mice were injected intramuscular with Rg1 at 20.0 $\mathrm{mg} / \mathrm{kg}$.bw and subsequent oral administration of SA at $10.0 \mathrm{mg} / \mathrm{kg} . b \mathrm{~b}$ once every other day for 14 days, renal tissues showed glomerular capillaries were mildly congested and no enlarged balloon, renal tubular epithelial cells were mildly edema, no obvious cell necrosis, and renal interstitium showed mildly edema (as shown by the black arrow); (IV) Rg1 treatment group: The morphology of renal tissues were normal, which was similar to control group, showed as normal shapes of glomerulus and renal tubules, the lumen of the renal tubules is regular, and no abnormality is observed in renal interstitium (as shown by the black arrow). 
A
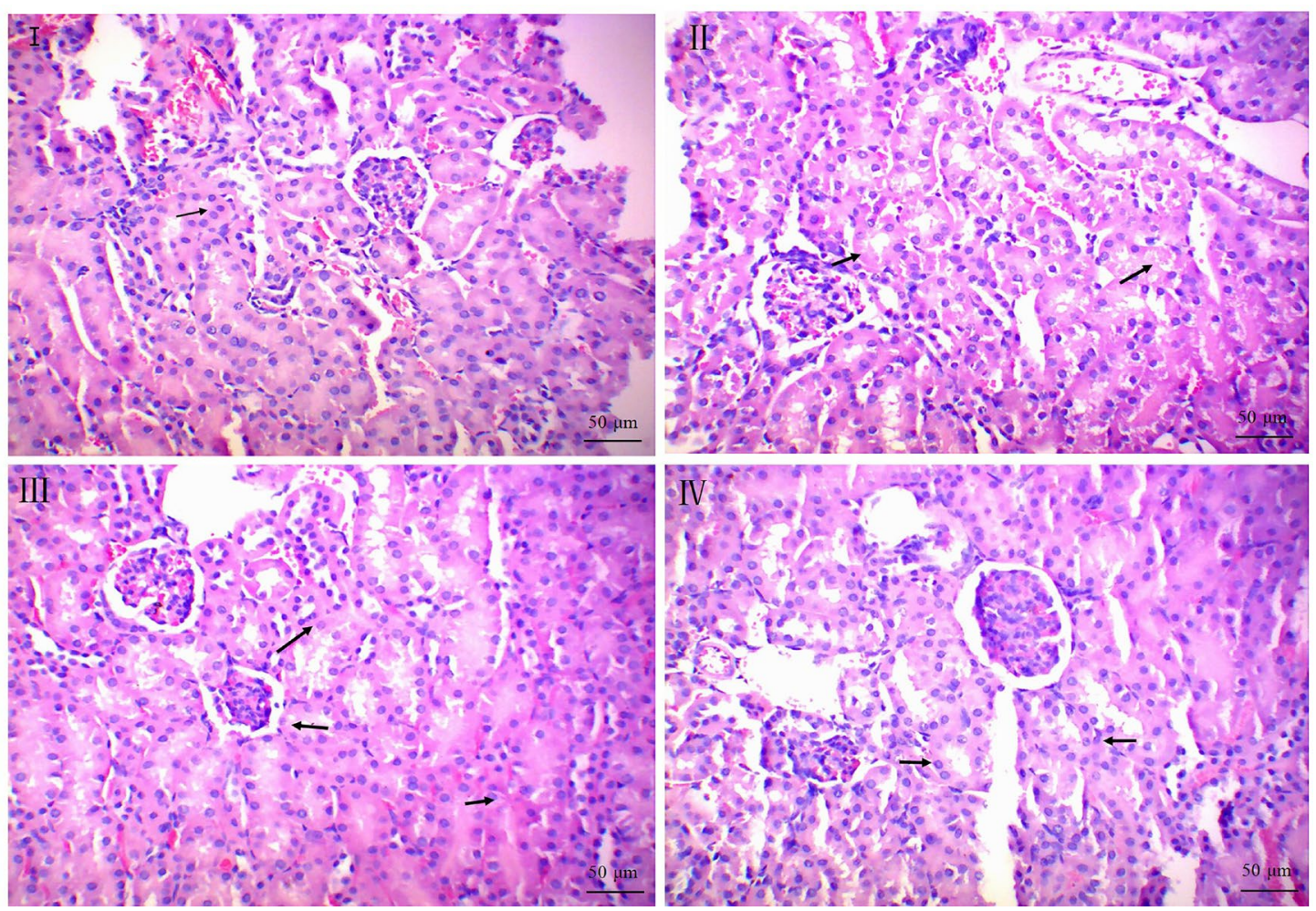

B

- Creatinine $(\mathrm{nmol} / \mathrm{ml})$

$\square$ TI score (\%)

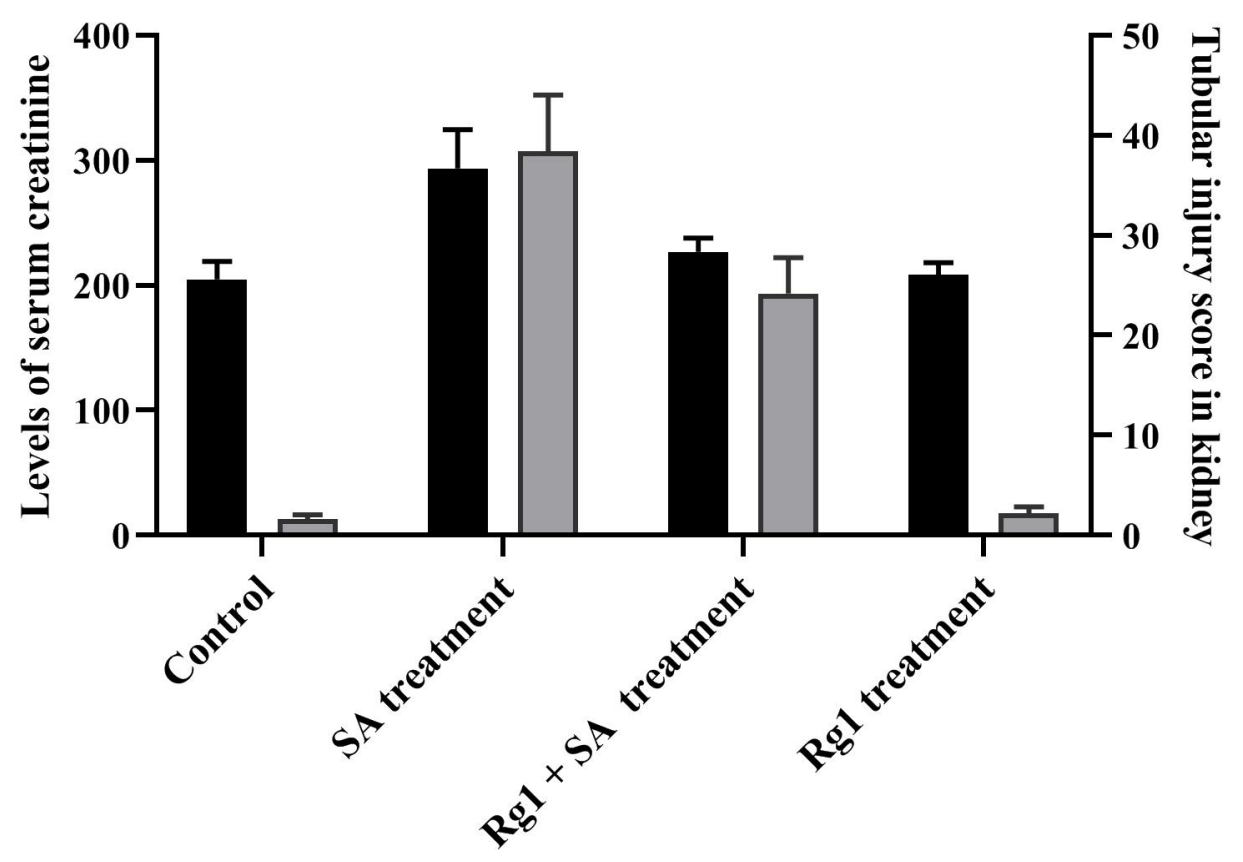


Figure 2. Levels of apoptosis, autophagy after SA treatment or Rg1 intervention

A. Histogram of renal apoptosis after SA treatment or Rg1 intervention. SA treatment showed the increased apoptosis in renal tissues, which was ameliorated significantly after $\operatorname{Rg} 1$ intervention; B. Representative images of TUNEL staining in renal tissues (x200). As shown by black arrow: Normal renal cells, shaped as regular round, cytoplasm showed blue or light blue staining; As shown by black arrow: TUNEL-positive cells showed chromatin condensation with color variation from yellow to dark brown, with irregular shapes and varied sizes. ( I ) Control group: Normal kidney cells, few apoptotic cells were found; (II) SA treatment group: The propotion of apoptotic cells was higher than that of control group; (III) Rg1 intervention+SA treatment group: The degree of apoptosis showed an alleviated characteristics compared to SA treatment group. (IV) Single Rg1 treatment group: The histological characteristics was similar to control group. C. Histogram of Beclin-1 or Caspase-3 positive percentages by IHC staining after SA exposure or Rg1 intervention. An increased Beclin-1 positive percentage and a decreased Caspase-3 positive percentage in kidney tissues were observed after SA treatment, which were antagonized by the intervention with Rg1. D. Representative immunofluorescence images of autophagy biomarker LC3-B by the confocal microscope $(\mathrm{x} 400)$. As shown by white arrows, blue staining represents cell nucleus, and the red staining spots around the nucleus indicate the autophagy marker LC3-B. ( I ) Control group, normal immunofluorescence of LC3-B staining; (II) SA exposure group, The enhanced LC3-B staining were observed in renal tissues; (III) Rg1 intervention + SA treatment group, a ameliorated immunofluorescence of LC3-B staining was observed in renal tissues; (IV) Rg1 treatment control, similar to control group.

Notes: Levels of TUNEL and IHC staining in each group are presented as mean percentage (\%), calculated by TUNEL or IHC-positive ratio of 10 fields under high power vision (magnification, $\mathrm{x} 200$ ). The sample size was defined as 1000 , and the significant difference between groups was obtained by $\chi^{2}$ test. ${ }^{*} P<0.05$ indicates a significant difference compared with control group; ${ }^{\#} P<0.05$ indicates a significant difference compared with SA treatment group.

Abbreviations: SA, sodium arsenite or $\mathrm{NaAsO}_{2}$; Rg1, Ginsenoside Rg1; TUNEL, Terminal Deoxynucleotidyl Transferase (TdT)-mediated deoxyuridine triphosphate (dUTP) Nick-End Labeling; IHC, immunohistochemical; LC3-B, microtubule-associated protein 1 light chain-3B. 
A

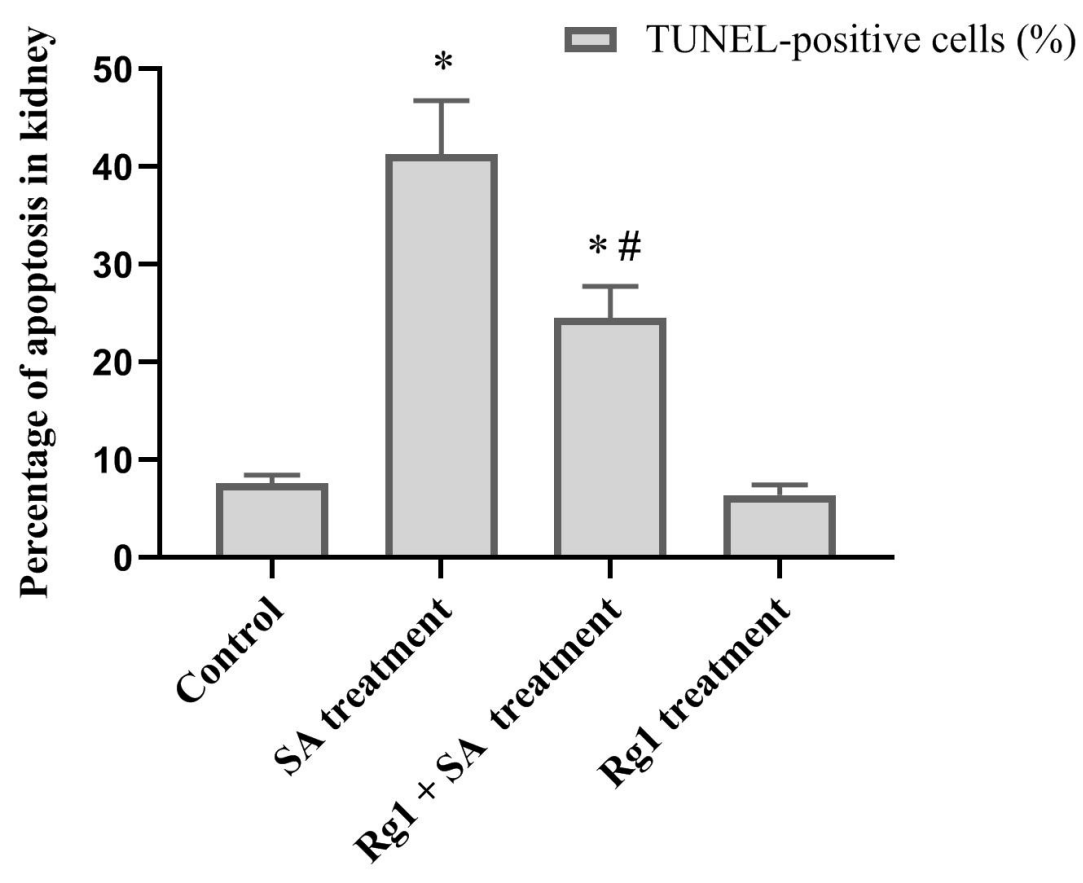

B

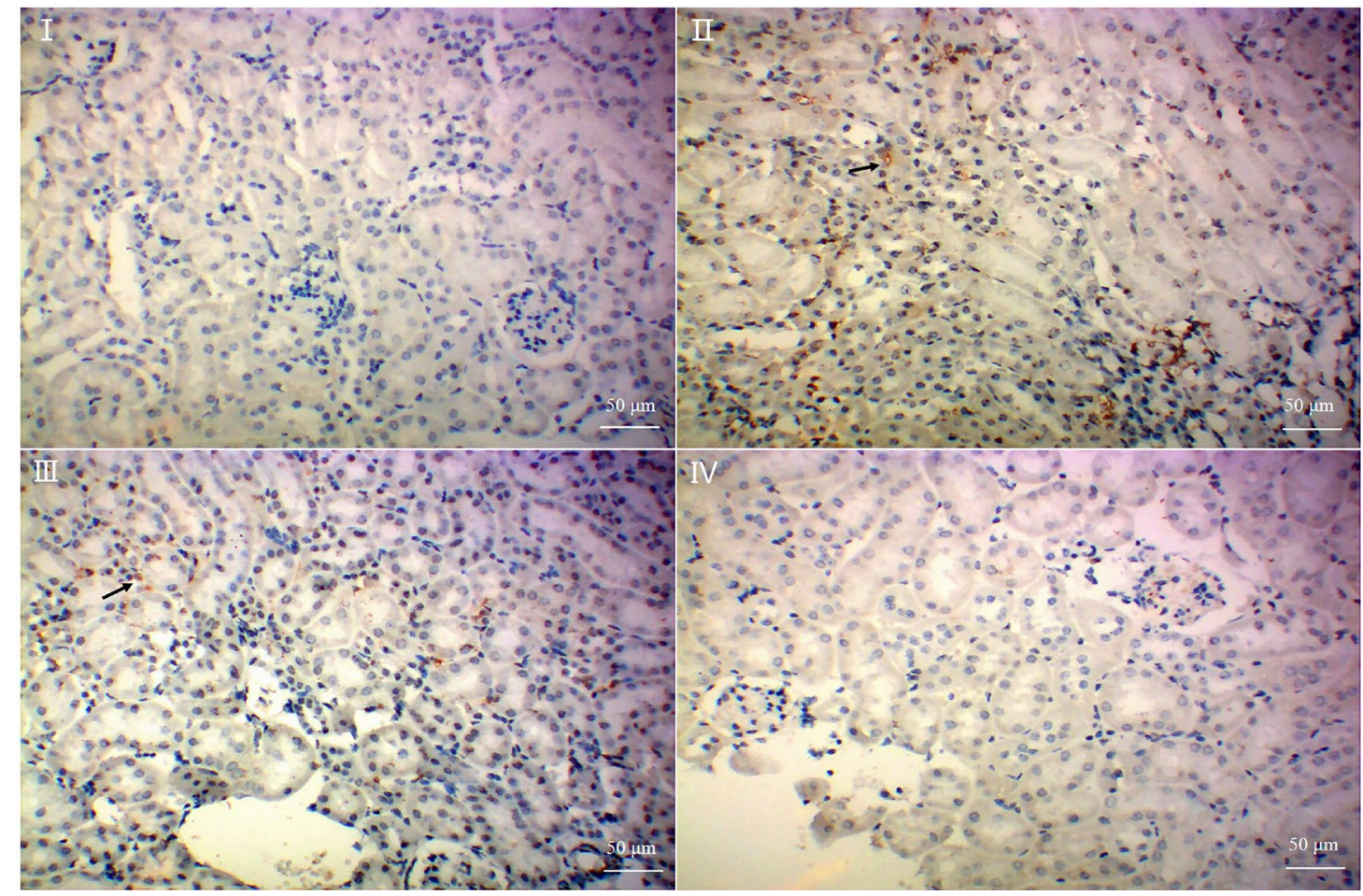


C

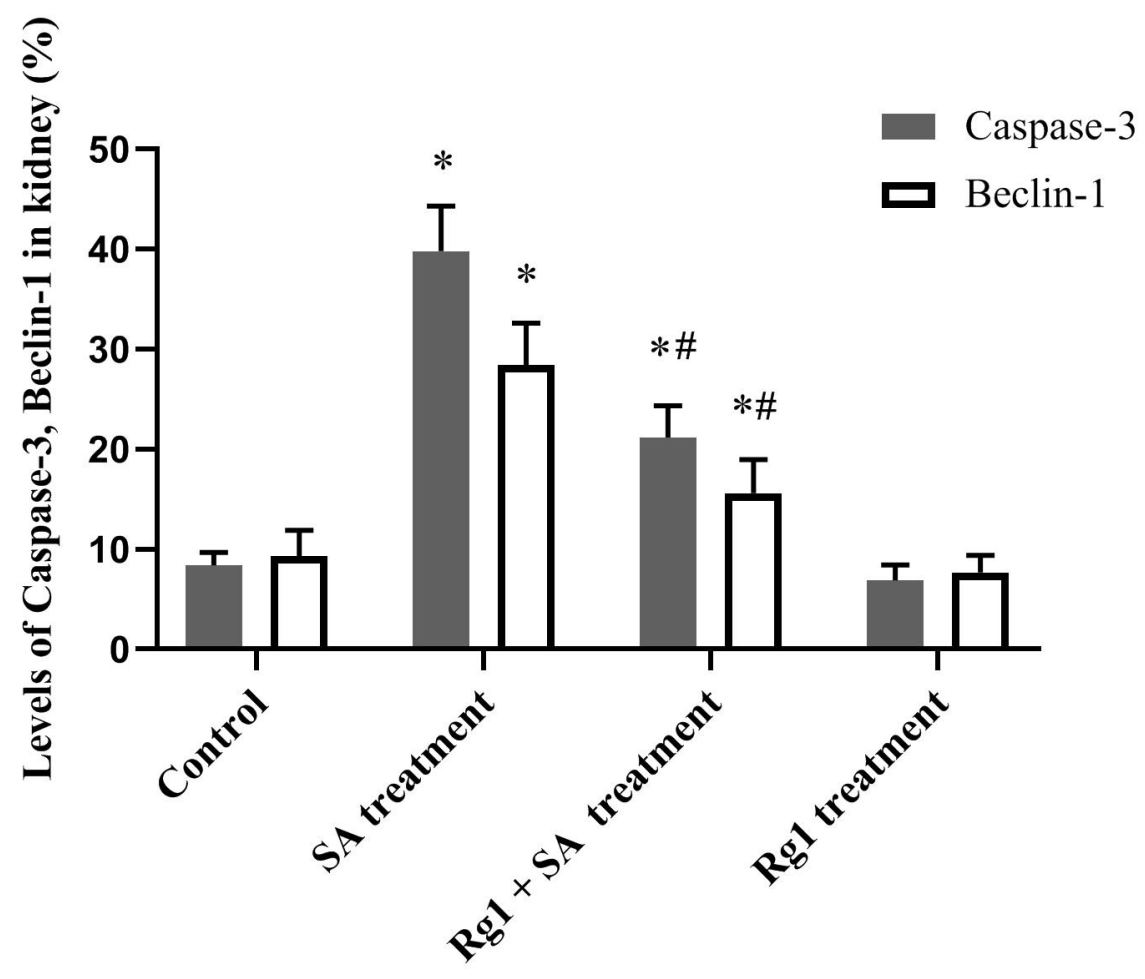

D

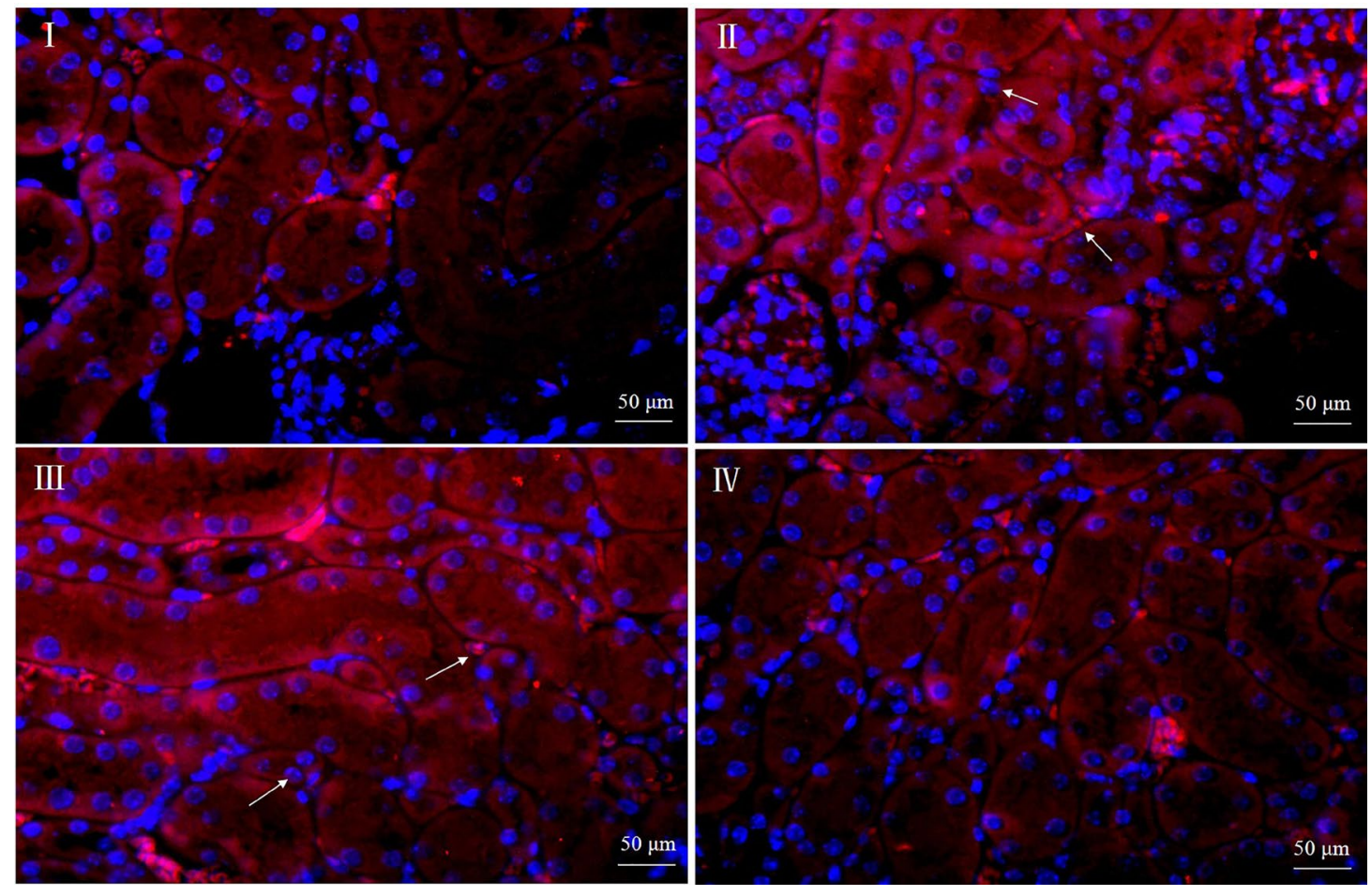


Figure 3. Levels of GSH, MDA after SA treatment or Rg1 intervention

Histogram of GSH, MDA after SA treatment or Rg1 intervention: a reduced GSH and a increased MDA were revealed in SA treatment group, which were antagonized by $\operatorname{Rg} 1$ intervention;

Notes: Data were expressed as mean $\pm \mathrm{SD}$; $\mathrm{n}=5$. the levels of GSH, MDA in kidney were quantified by nmol or ng values in per gram renal tissue according to the standard curve. The significant difference between groups was obtained by $\mathrm{T}$ tests followed ANOVA, ${ }^{*} P<0.05$ indicates a significant difference compared with control group, ${ }^{\sharp} P<0.05$ indicates a significant difference compared with SA treatment group. Abbreviations: GSH, glutathione; MDA, malondialdehyde; SD, standard deviation; ANOVA, one-way analysis of variance.

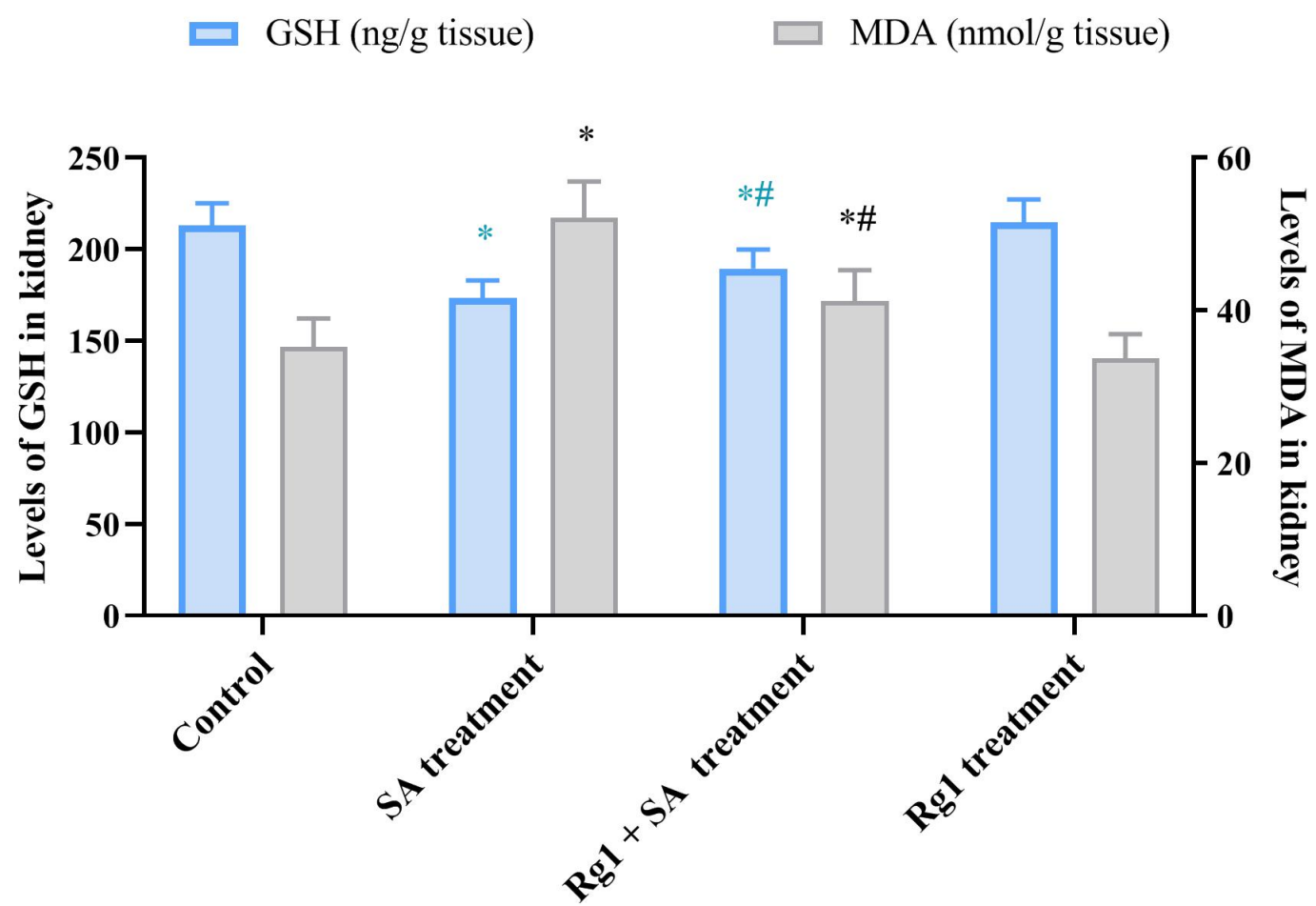


Figure 4. Characteristics of HO-1, mTOR, apoptosis or autophagy related signals in kidney

A. Levels of HO-1, mTOR and Bax in kidney after SA exposure or Rg1 intervention;

B. Levels of ULK1, SQSTM1/p62 in kidney after SA exposure or Rg1 intervention;

C. Levels of and LC3-A/B in kidney after SA exposure or Rg1 intervention.

The levels of HO-1, mTOR and SQSTM1/p62 showed a significant downregulation in SA exposure group compared to control group, while the intervention with Rg1 showed the upregulation of HO-1, mTOR and the recovery of SQSTM1. Conversely, the levels of Bax, ULK1, and LC3-B showed a significant elevation in SA exposure group compared to control group, while were downregulated after the intervention with $\operatorname{Rg} 1$.

Notes: Data were expressed as mean $\pm S D ; n=5$. Levels of proteins were evaluated by the measurement of relative integrated absorbance (IA), quantified by the ratio of detected protein vs. internal control ( $\beta$-actin) in each group (IA/IA). The significant difference between groups was obtained by $\mathrm{T}$ test followed ANOVA. ${ }^{*} P<0.05$ indicates a significant difference compared with control group; ${ }^{\#} P<0.05$ indicates a significant difference compared with SA treatment group. Abbreviations: HO-1, heme oxygenase-1; mTOR, mammalian target of rapamycin; Bax, Bcl-2 Associated X Protein; p62/SQSTM1, Sequestosome 1; ULK1, unc-51-like kinase-1; LC3-B, microtubule-associated protein 1 light chain-3B; IA, integrated absorbance. 
A

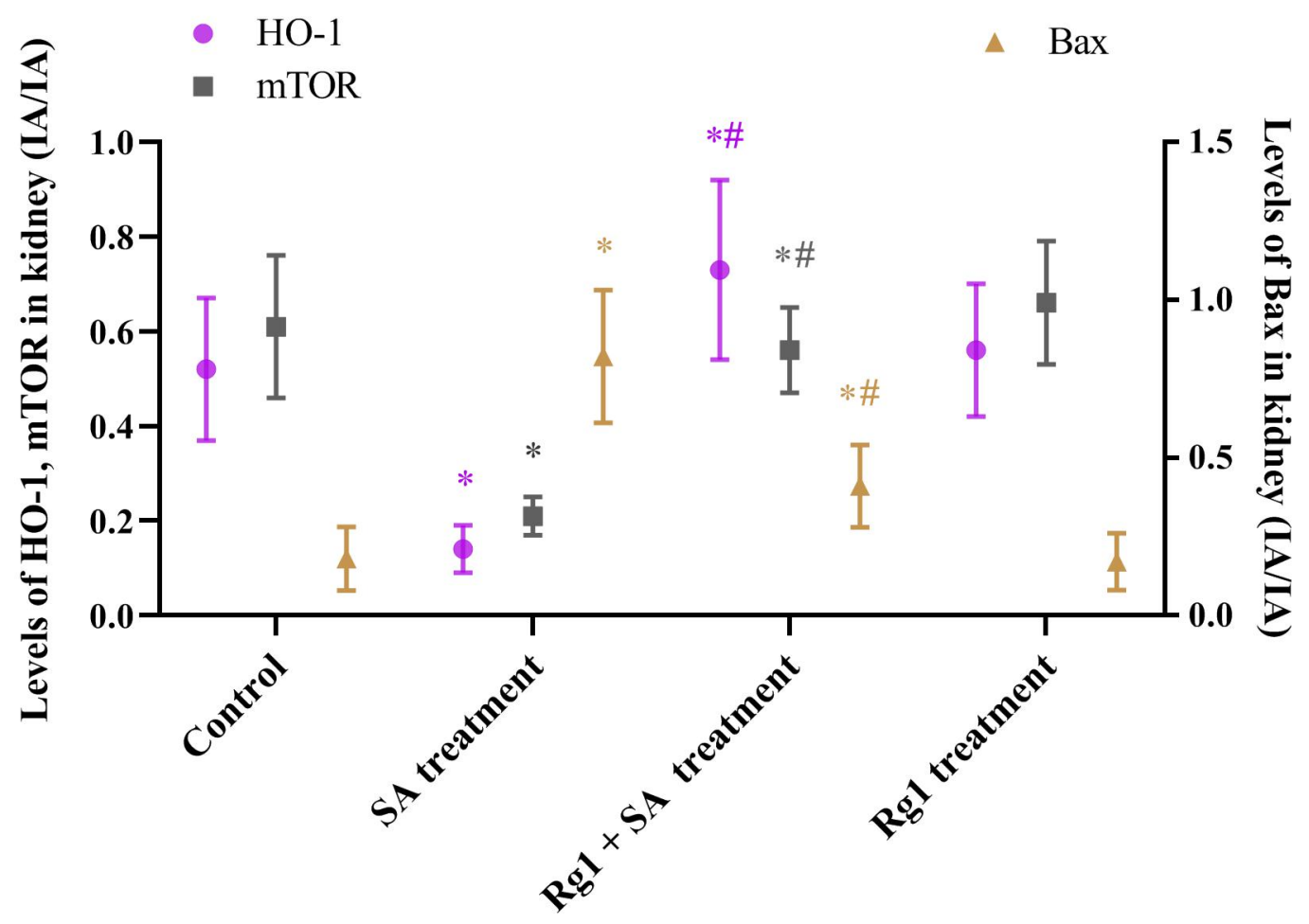

B

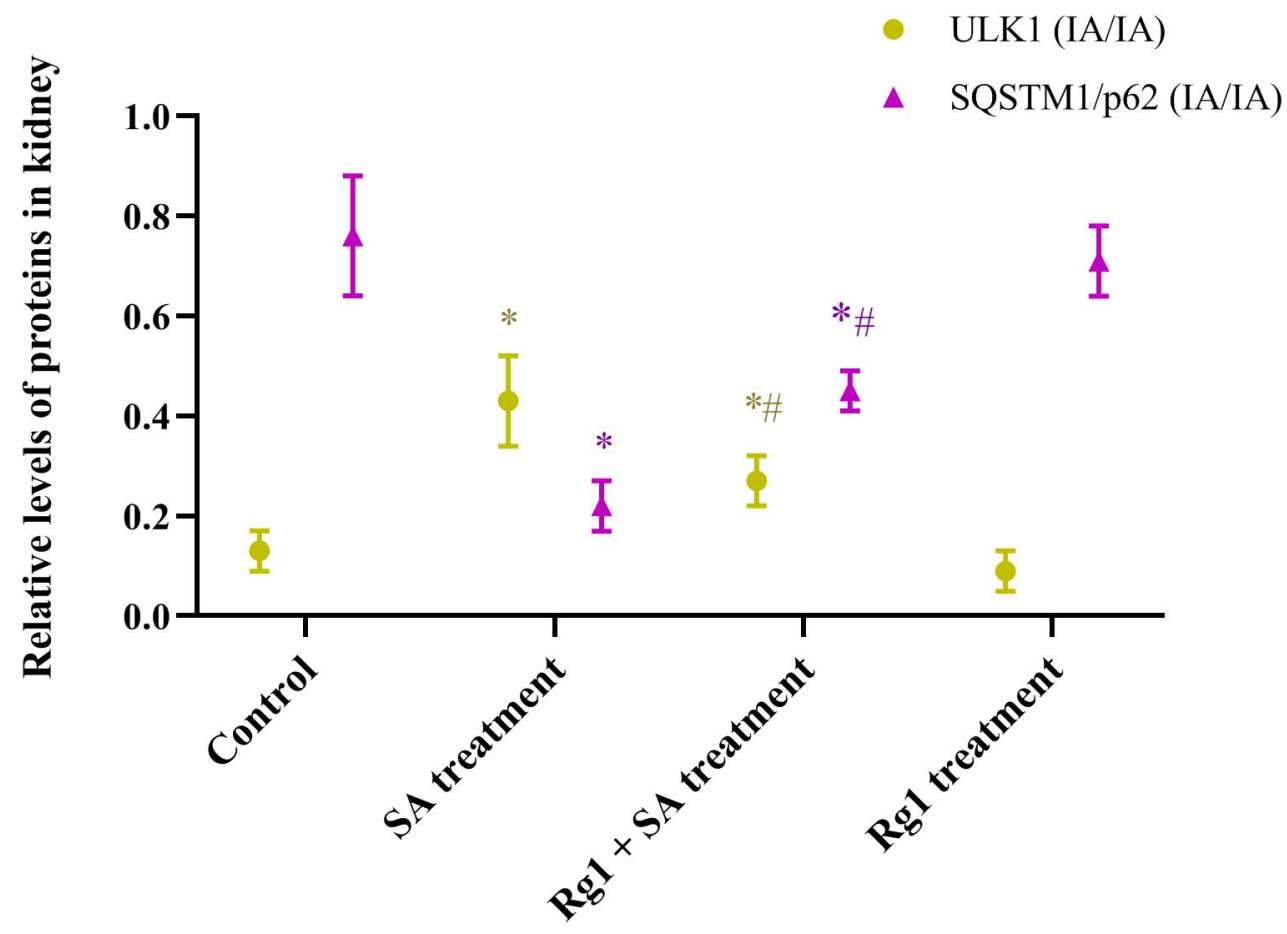


C

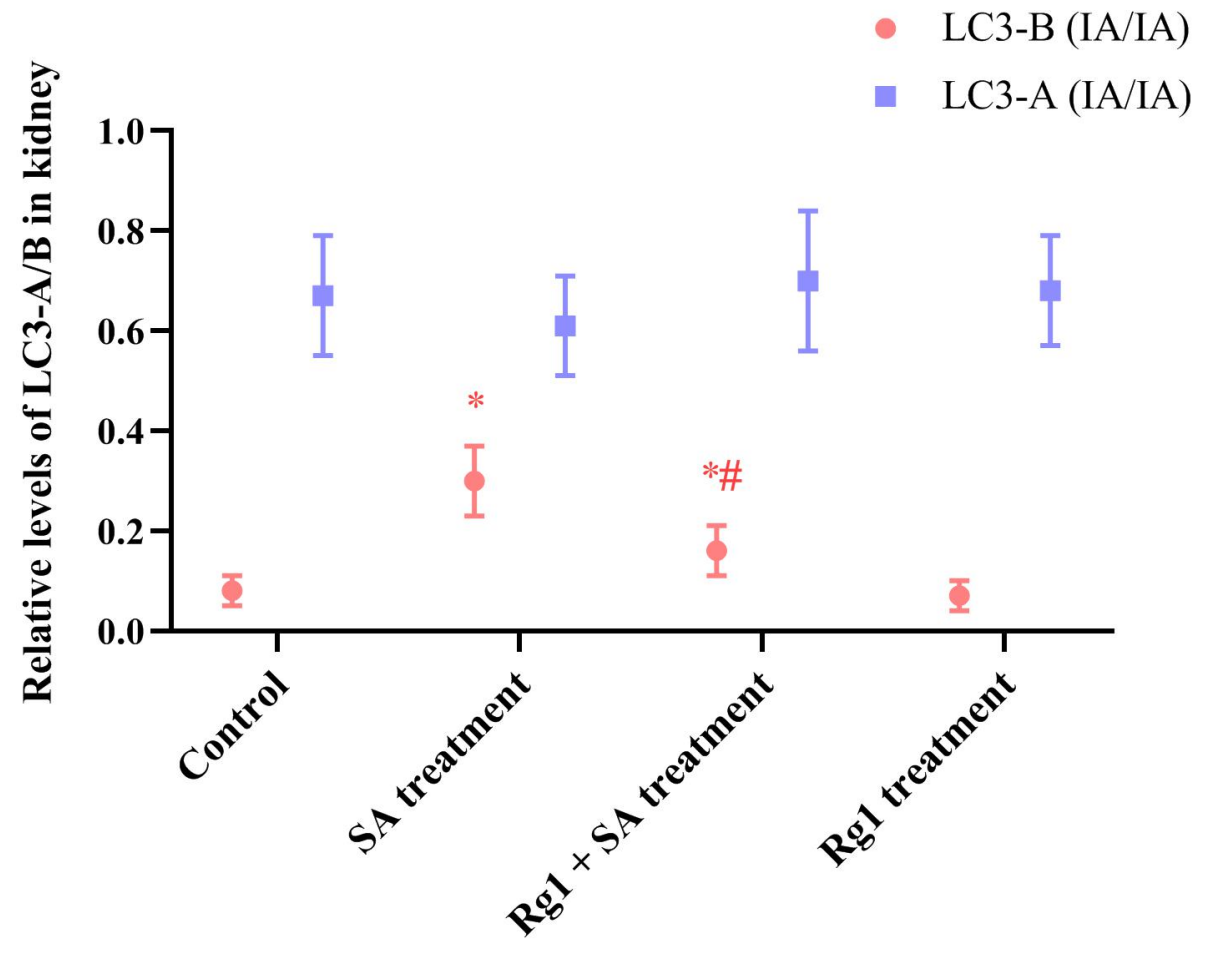




\section{Supplemental materials}

\section{Supplemental Figures}

Figure S1. Representative IHC staining images of Beclin-1 in renal tissues after SA treatment or Rg1 intervention.
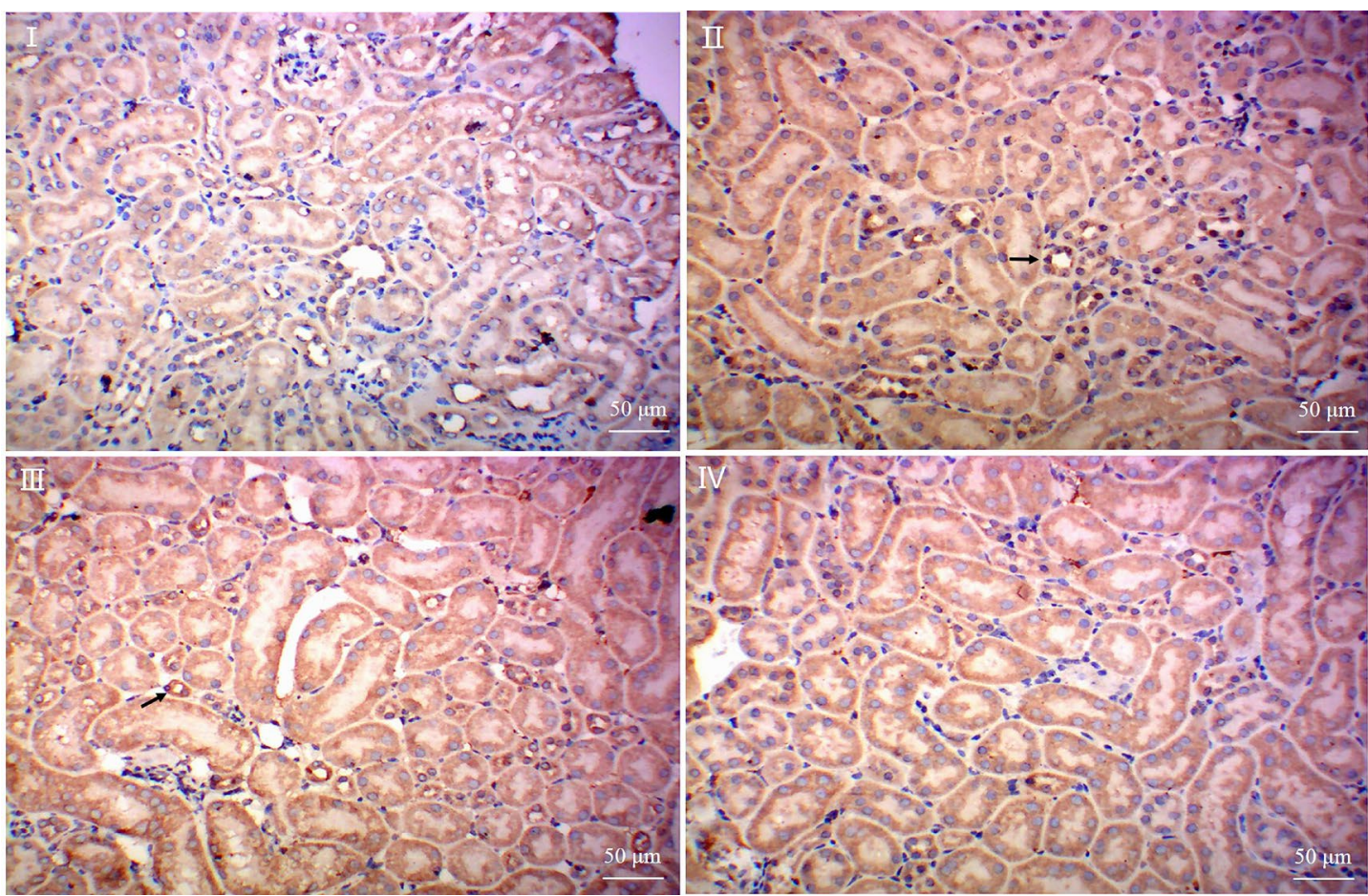
Figure S2. Representative IHC staining images of Caspase-3 in renal tissues after SA treatment or $\operatorname{Rg} 1$ intervention.
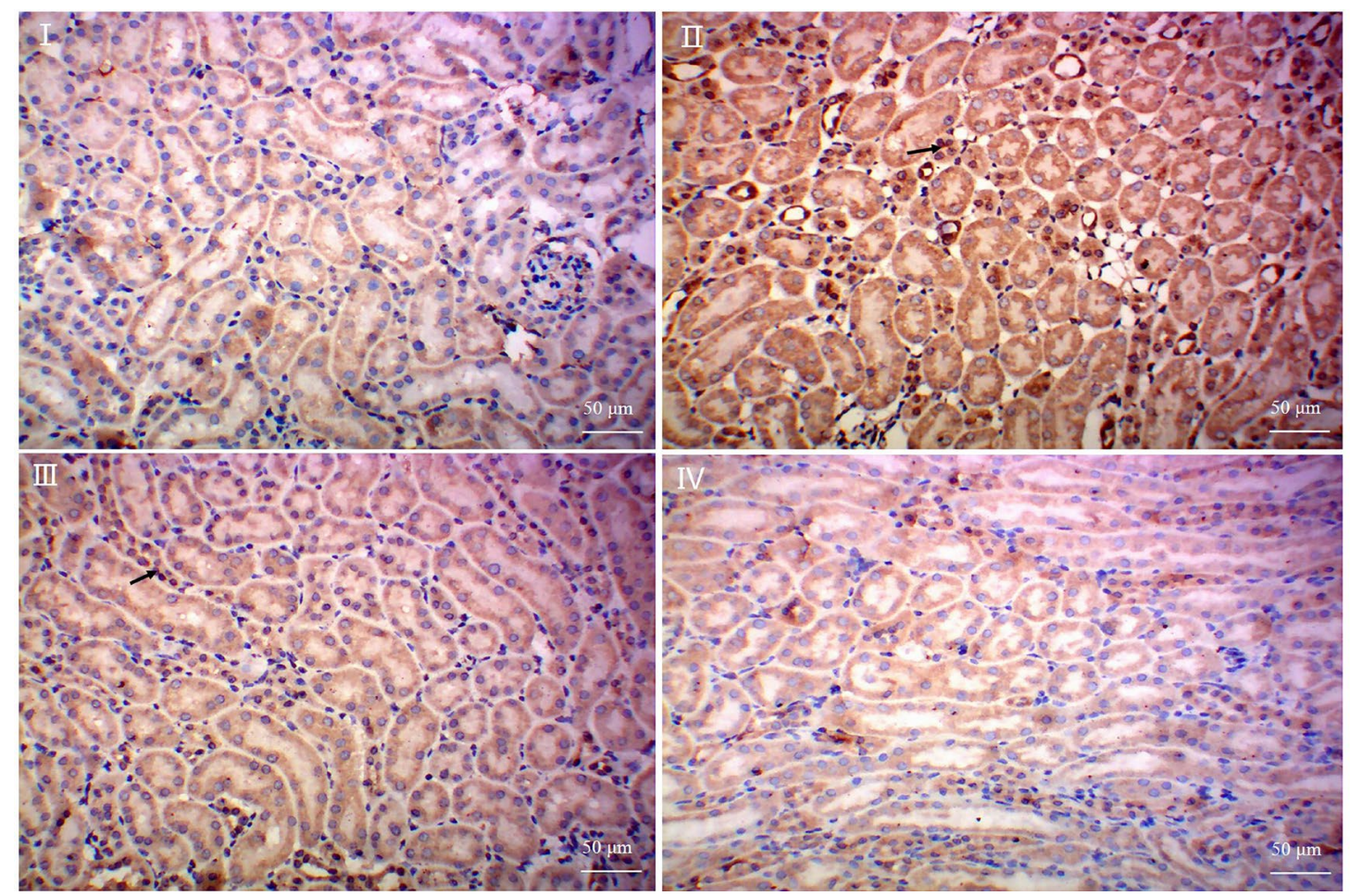

Abbreviations: SA, sodium arsenite; Rg1, Ginsenoside Rg1; IHC, immunohistochemical. 
Figure S3. Representative images of HO-1, mTOR, Bax by Western blotting analysis. Lane: 1. Control group; 2. SA treatment group; 3. Rg1 intervention + SA treatment group; 4. Rg1 treatment control.

\section{WB: Renal tissue}

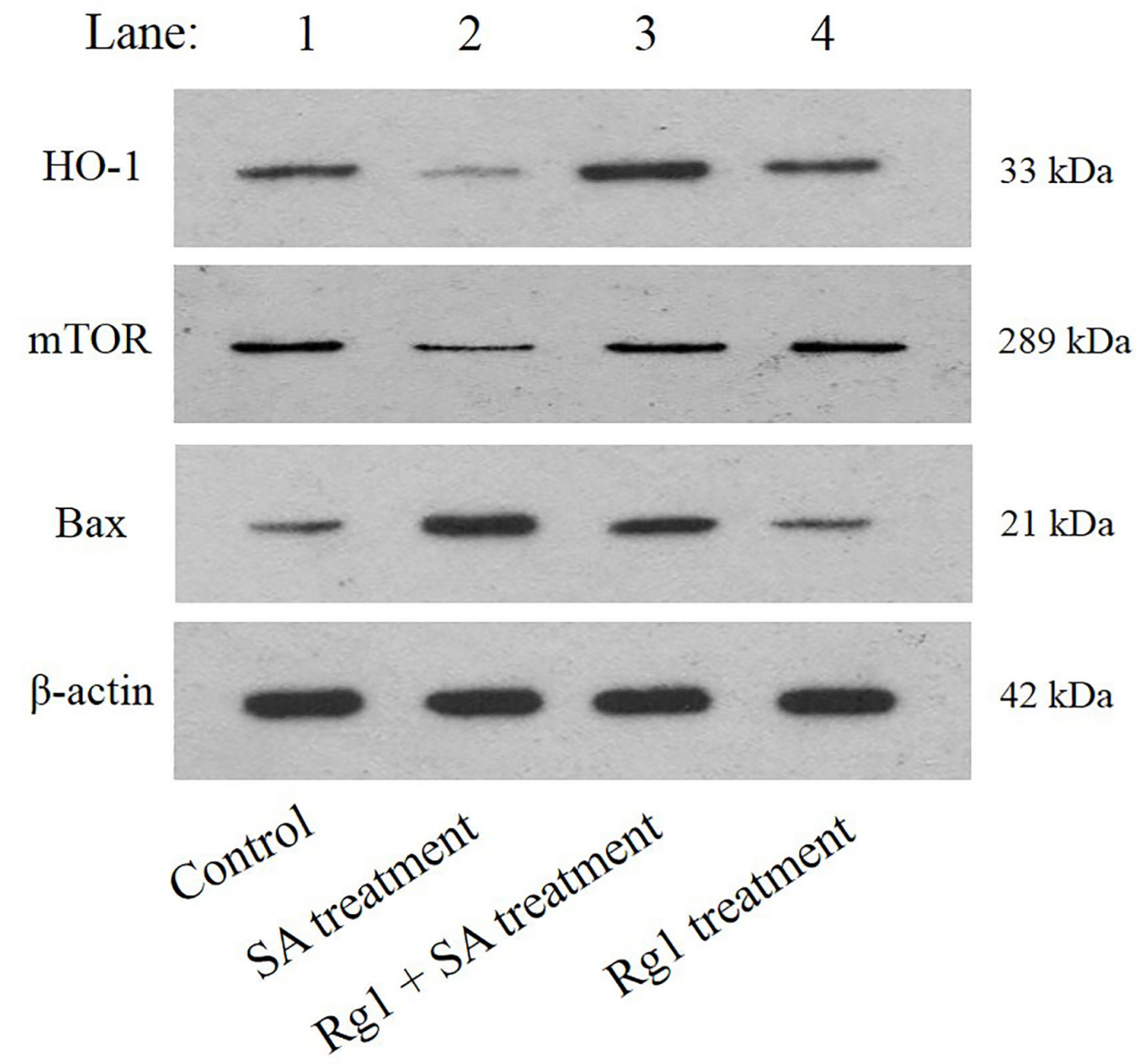

Abbreviations: SA, sodium arsenite; Rg1, Ginsenoside Rg1; Bax, Bcl-2 Associated X Protein; HO-1, Heme Oxygenase 1; mTOR, mammalian target of rapamycin. 
Figure S4. Representative images of SQSTM1/p62, ULK1, and LC3-A/B western blotting analysis from renal tissues in mice. Lane: 1. Control group; 2. SA treatment group; 3. Rg1 intervention + SA treatment group; 4. Rg1 treatment control.

\section{WB: Renal tissue}

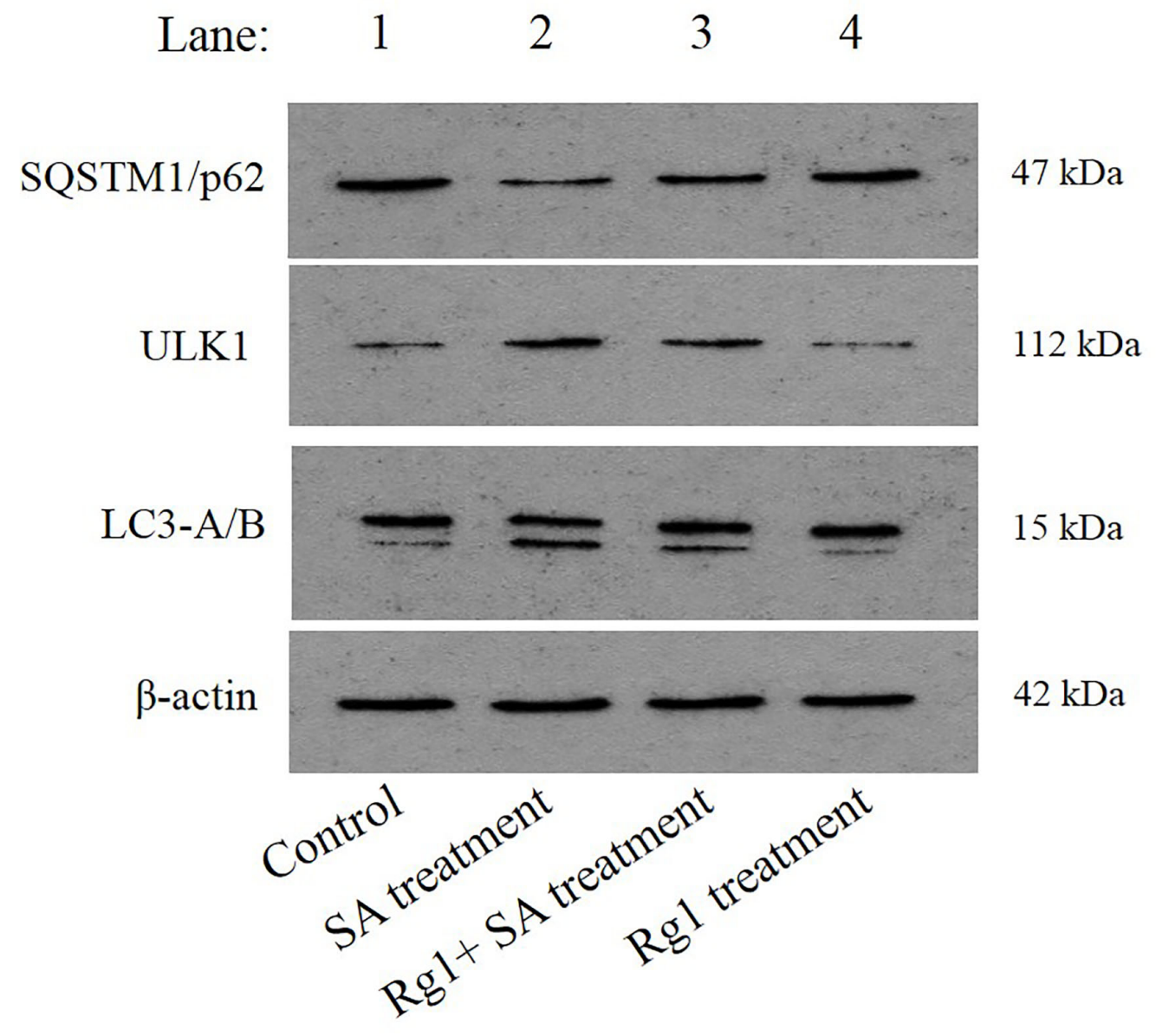

Abbreviations: SA, sodium arsenite; Rg1, Ginsenoside Rg1; p62/SQSTM1, Sequestosome 1; ULK1, unc-51-like kinase-1; LC3-A/B, microtubule-associated protein 1 light chain-3A/B. 


\section{Supplemental Tables}

Experimental dose of sodium arsenite (SA) was $10.0 \mathrm{mg} / \mathrm{kg} . \mathrm{bw}$ by oral administration once every other day in the period of 14 days, and the dose of $\operatorname{Rg} 1$ intervention was $20.0 \mathrm{mg} / \mathrm{kg} . \mathrm{bw}$ by intraperitoneal injection.

Table S1. Characteristics of Cr, KI score, TUNEL or IHC staining in renal sections

\begin{tabular}{lcccc}
\hline Indices (\%) & Control & SA treatment & Rg1+SA treatment & Rg1 treatment \\
\hline Scr & $204.86+14.47$ & $293.60+30.94^{*}$ & $226.95+10.87^{* \#}$ & $208.38+9.74$ \\
TI score & $1.6 \pm 0.48$ & $38.4 \pm 5.62^{*}$ & $24.2 \pm 3.56^{* \#}$ & $2.2 \pm 0.64$ \\
TUNEL ${ }^{+}$cells & $7.6 \pm 0.85$ & $41.3 \pm 5.48^{*}$ & $24.5 \pm 3.27^{* \#}$ & $6.4 \pm 1.02$ \\
Beclin-1 & $9.3 \pm 2.61$ & $28.4^{*} \pm 4.20$ & $15.6^{* \#} \pm 3.38$ & $7.7 \pm 1.71$ \\
Caspase-3 & $8.4 \pm 1.30$ & $39.8 \pm 4.52^{*}$ & $21.2 \pm 3.14^{* \#}$ & $6.9 \pm 1.53$ \\
\hline
\end{tabular}

Data were expressed as mean $\pm \mathrm{SD} ; \mathrm{n}=5$; Levels of TI score, TUNEL or IHC staining in each group were presented as mean percentage $(\%) \pm \mathrm{SD} ; \mathrm{n}=5$, calculated by TI, TUNEL or IHC-positive ratio of 10 fields under high power vision (magnification, x200). The significant difference between groups was obtained by $\chi^{2}$ test. ${ }^{*} P<0.05$ indicates a significant difference compared with control group; ${ }^{\#} P<0.05$ indicates a significant difference compared with SA treatment group.

Abbreviations: SA, sodium arsenite or $\mathrm{NaAsO}_{2}$; $\mathrm{Rg} 1$, Ginsenoside Rg1; Scr, serum creatinine; TI score, Tubular injury score in kidney; TUNEL ${ }^{+}$cells, Terminal Deoxynucleotidyl Transferase (TdT)-mediated deoxyuridine triphosphate (dUTP) Nick-End Labeling positive cells; IHC, immunohistochemical. 
Table S2. Characteristics of oxidative stress in renal sections after Rg1 intervention

\begin{tabular}{lcccc}
\hline Indices (Units) & Control & SA treatment & Rg1+SAtreatment & Rg1 treatment \\
\hline $\begin{array}{l}\text { MDA (nmol/g } \\
\text { tissue) }\end{array}$ & $35.18+3.74$ & $52.12+4.75^{*}$ & $41.24+4.04^{* \#}$ & $33.67+3.18$ \\
& & & & \\
& & & & \\
GSH (ng/g tissue) & $213.07+12.08$ & $173.34+9.73^{*}$ & $189.54+10.40^{* \#}$ & $214.76+12.35$ \\
\end{tabular}

Data were expressed as mean $\pm \mathrm{SD} ; \mathrm{n}=5$. the levels of GSH, MDA in kidney were quantified by nmol or ng values in per gram renal tissue according to the standard curve. The significant difference between groups was obtained by $\mathrm{T}$ test followed ANOVA, ${ }^{*} P<0.05$ indicates a significant difference compared with control group, ${ }^{\#} P<0.05$ indicates a significant difference compared with SA treatment group. Abbreviations: MDA, malondialdehyde; GSH, glutathione; $\mathrm{SD}$, standard deviation; ANOVA, one-way analysis of variance. 
Table S3. Characteristics of HO-1, mTOR and apoptosis or autophagy-associated signals in kidney

\begin{tabular}{lcccc}
\hline Indices (IA/IA) & Control & SA treatment & Rg1 + SA treatment & Rg1 treatment \\
\hline HO-1 & $0.52+0.15$ & $0.14+0.05^{*}$ & $0.73+0.19^{* \#}$ & $0.56+0.13$ \\
mTOR & $0.48+0.13$ & $0.21+0.07^{*}$ & $0.54+0.11^{\#}$ & $0.56+0.15$ \\
Bax & $0.18+0.10$ & $0.82+0.21^{*}$ & $0.41+0.13^{* \#}$ & $0.17+0.09$ \\
SQSTM1/p62 & $0.76+0.12$ & $0.22+0.05^{*}$ & $0.45+0.04^{* \#}$ & $0.71+0.07$ \\
ULK1 & $0.13+0.04$ & $0.43+0.09^{*}$ & $0.27+0.05^{* \#}$ & $0.09+0.04$ \\
LC3-A & $0.67+0.12$ & $0.61+0.10$ & $0.70+0.14$ & $0.68+0.11$ \\
LC3-B & $0.08+0.03$ & $0.30+0.07^{*}$ & $0.16+0.05^{* \#}$ & $0.07+0.03$ \\
\hline
\end{tabular}

Data were expressed as mean $\pm \mathrm{SD} ; \mathrm{n}=5$. Levels of proteins were evaluated by the measurement of relative level of IA, quantified by the ratio of detected protein vs. internal control ( $\beta$-actin) in each group (IA/IA). The significant difference between groups was obtained by $\mathrm{T}$ test followed ANOVA. ${ }^{*} P<0.05$ indicates a significant difference compared with control group; ${ }^{\#} P<0.05$ indicates a significant difference compared with SA treatment group.

Abbreviations: WB, Western-Blotting; HO-1, Heme Oxygenase 1; mTOR, mammalian target of rapamycin; Bax, Bcl-2 Associated X Protein; p62/SQSTM1, Sequestosome 1; ULK1, unc-51-like kinase-1; LC3-A/B, microtubule-associated protein 1 light chain-3A/B; IA, integrated absorbance. 